\title{
1 Generation of multi-color carrier-envelope phase locked pulse with continuous color tunability
}

\author{
Atsushi Yabushita, ${ }^{1, *}$ Ding-Yi Juang, ${ }^{1}$ Chih-Hsien Kao, ${ }^{1}$ Andrius Baltuška, ${ }^{2}$ and \\ Takayoshi Kobayashi ${ }^{1,3,4,5}$ \\ ${ }^{1}$ Department of Electrophysics, National Chiao-Tung University, Hsinchu 300, Taiwan \\ ${ }^{2}$ Institut für Photonik, Technische Universität Wien, Wien 1040, Austria \\ ${ }^{3}$ Department of Applied Physics and Chemistry and Institute for Laser Science, University of Electro- \\ Communications, Chofu, Tokyo 182-8585, Japan \\ ${ }^{4}$ Core Research for Evolutional Science and Technology, Japan Science and Technology Agency, Tokyo, 102-0076 \\ Japan \\ ${ }^{5}$ Institute of Laser Engineering, Osaka University, Suita, Osaka 565-0971, Japan \\ “yabushita@mail.nctu.edu.tw
}

\begin{abstract}
Several methods exist to lock carrier envelope phase (CEP), which is generally not locked in common laser systems. Our previous work succeeded passive lock of CEP by using a technique of non-collinear optical parametric amplifier. In the present work, we have proposed and demonstrated two schemes to generate multi-color CEP-locked beams using the non-collinear optical parametric amplifier. In the first scheme, spatial filter in the spatially dispersed seed light was used to generate three-color CEP-locked pulse demonstrating its arbitral color tunability. In the second scheme, parabolic chirp was introduced on the seed pulse to generate two color CEP-locked pulses with continuous color tunability.
\end{abstract}

keywords: Lasers, pulsed; Nonlinear optics, parametric processes.

\section{Introduction}

Carrier envelope phase (CEP) of the laser pulses are generally not locked in conventional laser systems. Meanwhile, CEP-locked laser pulse has various applications such as soft X-ray generation [1], frequency metrology [2,3], and control of diatomic molecule dissociation [4-6]. Therefore, various methods have been developed to lock the CEP. These methods can be divided into two groups; active CEP-lock methods and passive CEP-lock methods.

In active CEP-lock methods $[2,7,8]$, the laser spectrum is broadened over one octave using non-linear medium like a microstructure optical fiber. The high frequency component of the broadened spectrum was mixed with the double frequency of the low-frequency component to observe their interference (f- $2 \mathrm{f}$ interferometer). The observed beat reflecting the change of CEP was used as (active) feed-back signal to stabilize the CEP of the laser.

Following that, we have developed another type of CEP-lock method called as a passive CEP-lock method [9] using a non-collinear optical parametric amplifier (NOPA) [10-16]. Second harmonic (SH) of the Ti:sapphire laser was used not only to pump the NOPA but also to seed the NOPA generating the white-light continuum generation. The CEP of the pump pulse randomly shifts its value pulse-to-pulse, but the amount of shift is equal to that of the seed pulse in the NOPA. Therefore, the idler pulse coming out from the NOPA is locked to a constant phase not experiencing any shift in pulse-to-pulse.

In the present work, we have developed and demonstrated two schemes to generate multicolor CEP-locked pulses. The CEPs of the pulses are self-stabilized by the NOPA. The seed pulse of the NOPA was spectral or temporally modified to generate multi-color CEP-locked pulse. 


\section{Experiment}

The broadband CEP-locked pulse was generated by a NOPA (see Fig. 1). As a light source of the NOPA, we have used a Ti:sapphire regenerative amplifier (Legend-USP; Coherent Inc.) seeded with a Ti:sapphire laser oscillator (Micra-10; Coherent Inc.). The amplifier generates femtosecond pulses with 40fs (duration), $800 \mathrm{~nm}$ (central wavelength), $5 \mathrm{kHz}$ (repetition rate), and $2.5 \mathrm{~W}$ (average power). The pulse from the regenerative amplifier was focused into a $1 \mathrm{~mm}$-thick $\beta$-barium borate (BBO) crystal to generate second harmonic ( $\mathrm{SH}$ ) with average power of $200 \mathrm{~mW}$. When the SH power was increased higher than $200 \mathrm{~mW}$, the SH pulse generated self phase modulation in the NOPA crystal, which caused serious loss in the amplification gain of the NOPA. Therefore, the SH power was set to be $200 \mathrm{~mW}$. The $\mathrm{SH}$ pulse was separated into two pulses by a beam sampler (BSF10-A; thorlabs).

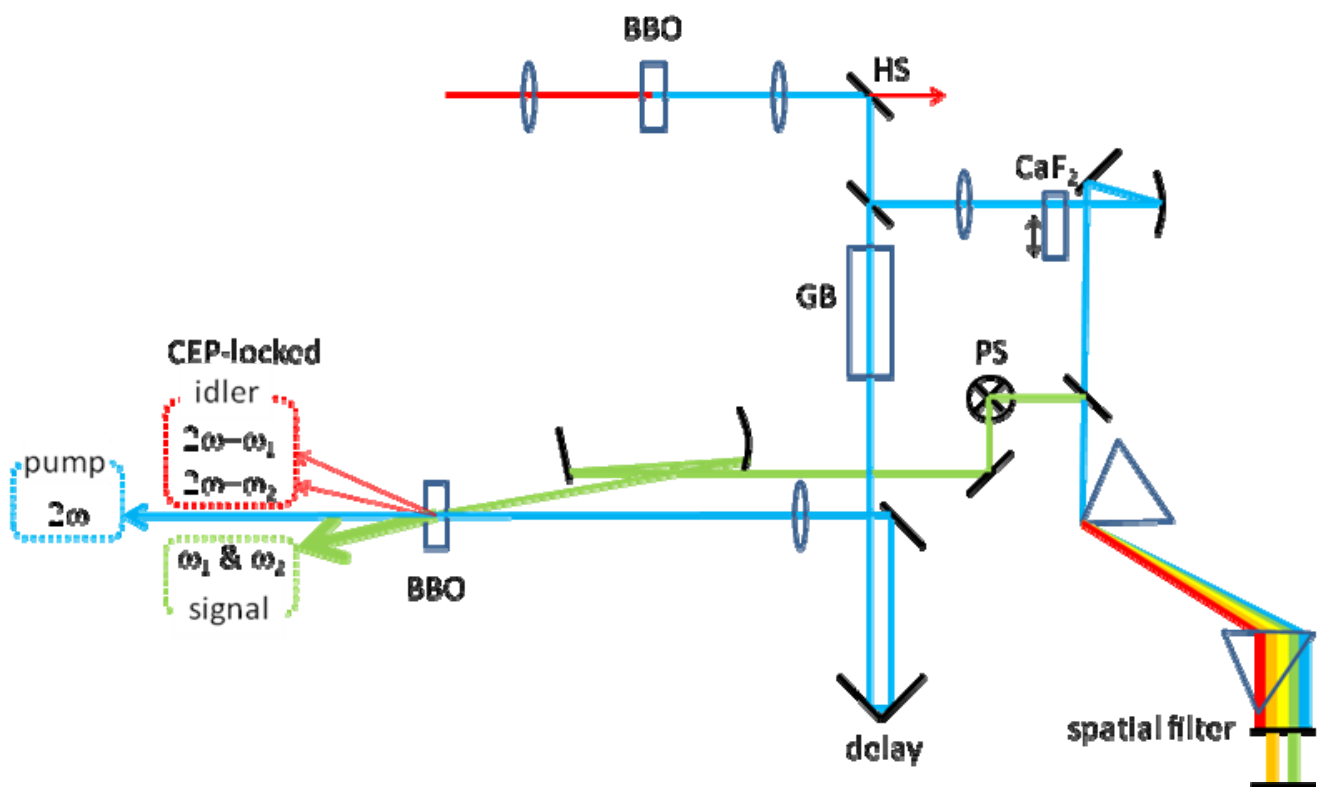

Fig. 1. Experimental setup to generate multi-color CEP-locked pulse. BBO: $\beta-\mathrm{BaB}_{2} \mathrm{O}_{4}$ crystal; .HS: harmonic separator; $\mathrm{GB}$ : quartz glass block, $\mathrm{CaF}_{2}: \mathrm{CaF}_{2}$ plate; PS: periscope; delay: manual translation stage with a micrometer.

About $1 \%$ of the SH pulse was reflected on the non-coated surface of the beam sampler. Then a lens focused the weak SH pulse into a calcium fluoride $\left(\mathrm{CaF}_{2}\right)$ plate to generate white light (WL) by self-phase modulation. The $\mathrm{CaF}_{2}$ plate was pasted on edge of a top plate of a mechanical rotation stage (SGSP-40YAW; sigma koki). The $\mathrm{CaF}_{2}$ plate was shaken vertical to the light path oscillating the set angle of the mechanical rotation stage in the range of $+/-1$ degree. The periodical transitional motion of the $\mathrm{CaF}_{2}$ plate was performed not to make a permanent damage on the plate under irradiation of the SH beam. The WL pulse generated from the SH pulse was used as a seed pulse of the NOPA after rotating the polarization by a periscope.

In the light path of the WL pulse, we have inserted a prism compressor, which consists of a pair of F2 equilateral dispersive prism (PS852 and PS854; thorlabs). The prism compressor had two functions in the present work. One is to compress the linear chirp of the WL seed pulse except for high order chirp. Large chirp at the spectrum edge of the WL seed pulse allowed us to generate double color pulse modulating the optical delay between the pump 
pulse and the seed pulse (see section 3-3). The other function of the prism compressor is for arbitrary spectral modulation in the NOPA seed spectrum, which could be performed inserting a spatial filter in the seed beam path spatially dispersed in the prism compressor.

About $98 \%$ of the SH pulse transmitted through the beam sampler. The intense SH beam stretched its pulse duration passing through a fused silica glass block with length of $40 \mathrm{~mm}$, and used as a pump pulse of the NOPA. The long duration ( 200fs) of the pump pulse avoids damage on the optical components and makes the NOPA output to be less sensitive to the timing drifts (between the pump pulse and the seed pulse). The tolerance to the timing drifts improves the power stability of the NOPA output.

The CEP of the fundamental pulse $\phi_{\text {fund }}$ from the regenerative amplifier is not locked, and the CEP of the SH pulse $\phi_{S H}=2 \phi_{\text {fund }}+\pi / 2$ is also not locked. The SH pulse was used as the NOPA pump pulse, whose CEP is $\phi_{\text {pump }}=\phi_{S H}$. The SH pulse was also used for generation of the NOPA seed pulse, whose CEP is $\phi_{\text {seed }}=\phi_{S H}+\pi / 2$. The CEP of the NOPA idler can be calculated as $\phi_{\text {idler }}=\phi_{\text {pump }}-\phi_{\text {seed }}-\pi / 2$ (see section 3.3 for derivation of this equation or ref. 21 for more detail of the derivation procedure). Therefore, the NOPA idler pulse has a constant CEP of $\phi_{\text {idler }}=\phi_{S H}-\left(\phi_{S H}+\pi / 2\right)-\pi / 2=-\pi$. Thus, the CEP of the NOPA idler pulse is free from the phase drift being self-stabilized at a constant value.

In our previous report, the stability of the CEP in a passive CEP stabilization system using NOPA was estimated as $\pm \pi / 10 \mathrm{rad}$ [9]. The passive CEP stabilization system used in the present work is same as the one used in the previous work, except for the prism pair newly inserted in the present work. The prism pair introduced wavelength tunability in the system, meanwhile the prism pair introduced high order chirp in the seed pulse narrowing the gain bandwidth. In the present setup, the narrowed bandwidth makes hard to estimate the CEP stability by f-to-2f interferometer, which requires one octave bandwidth. However, the identicalness of the setup between the previous work and the present work is thought to result in the same CEP stability of $\pm \pi / 10 \mathrm{rad}$.

\section{Results and discussion}

\subsection{Simulation of the chirp generated by a prism pair}

As a prism pair, we have used equilateral dispersive prisms made of $\mathrm{F} 2$ glass putting them in the minimum declination angle. The distance between the two apexes of the prism pair was $380 \mathrm{~mm}$. The refractive index of the F2 glass can be calculated as

$$
\mathrm{n}=\sqrt{1+\frac{\mathrm{B}_{1} \lambda^{2}}{\lambda^{2}-\mathrm{C}_{1}}+\frac{\mathrm{B}_{2} \lambda^{2}}{\lambda^{2}-\mathrm{C}_{2}}+\frac{\mathrm{B}_{3} \lambda^{2}}{\lambda^{2}-\mathrm{C}_{3}}}
$$
$\mathrm{C}_{2}=4.70450767 \times 10^{-2}, \mathrm{C}_{3}=1.11886764 \times 10^{2}$, and $\lambda$ is the wavelength of the light in unit of $\mu \mathrm{m}$. These parameter values are provided by SCHOTT North America. The ray trace of the light was calculated from $500 \mathrm{~nm}$ to $750 \mathrm{~nm}$ at $10 \mathrm{~nm}$ step as shown in Fig. 2a. 


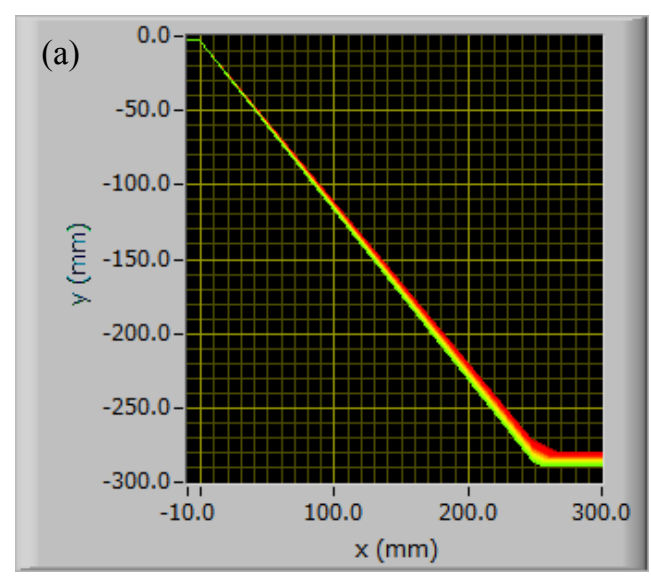

(c)

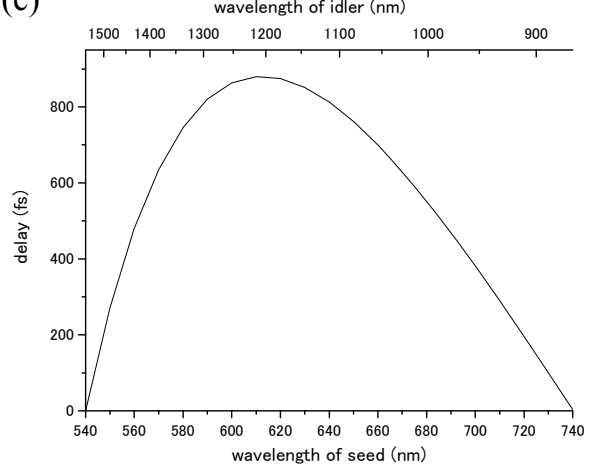

(b)

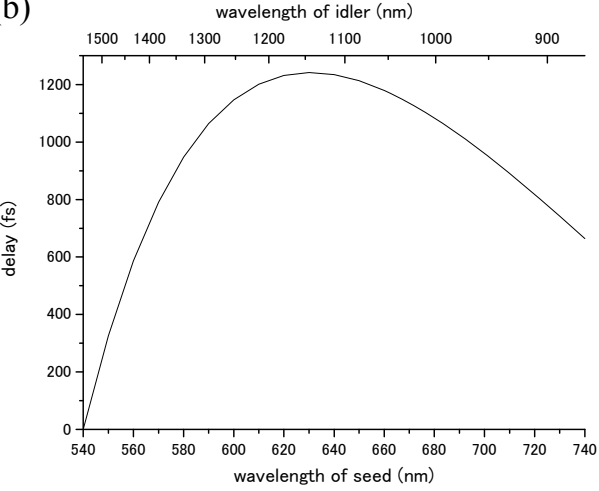

(d)

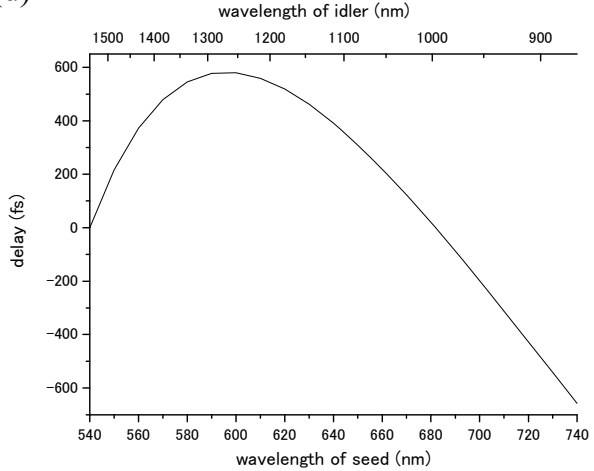

Fig. 2. (a) Ray trace of the light calculated in the spectral region from $500 \mathrm{~nm}$ to $750 \mathrm{~nm}$ at 10 $\mathrm{nm}$ step. Apexes of the prism pair were put at $(\mathrm{x}, \mathrm{y})=(0 \mathrm{~mm}, 0 \mathrm{~mm})$ and $(250 \mathrm{~mm},-292 \mathrm{~mm})$, and the input beam was assumed to be running from negative $x$ on $y=-2.0 \mathrm{~mm}$ with $\mathrm{x}$ axes parallel. The RGB (red, green, blue) values for each wavelength were calculated using Dan Bruton's algorithm and used to plot the ray trace for each wavelength. (b-d) Group delay of the seed pulse when the prism insertion amount was changed putting the apex of the prism at (b) (250 mm, -290 mm), (c) (250 mm, -292 mm), and (d) $(250 \mathrm{~mm},-294 \mathrm{~mm})$,

One of the ray tracing results is shown in Fig. 2a, putting apexes of the prism pair at $(x, y)$ $=(0 \mathrm{~mm}, 0 \mathrm{~mm})$ and $(250 \mathrm{~mm},-293 \mathrm{~mm})$. In the calculation the input beam was assumed to be running from negative $x$ on $y=-2.0 \mathrm{~mm}$ with $x$ axes parallel. The ray tracing curves were plotted using approximate RGB (red, green, blue) values for visible wavelengths, which were obtained by Dan Bruton's algorithm [17].

Group velocity of the light in a material can be calculated using the refractive index of the material, $n$, as $v_{g}=c /(n+\omega \cdot d n / d \omega)$. Using the equation, we have estimated the group delay dispersion (GDD) of the WL seed pulse added by the prism pair. We also calculated the GDD of the WL seed pulse experienced in transmission through the $\mathrm{CaF}_{2}$ plate after the WL seed pulse was generated in the plate. In calculation of the GDD caused by the $\mathrm{CaF}_{2}$ plate, we have used the refractive index of $\mathrm{CaF}_{2}$, which can also be calculated using Eq. (1) where $\mathrm{B}_{1}=5.675888 \times 10^{-1}, \mathrm{~B}_{2}=2.52643 \times 10^{-3}, \mathrm{~B}_{3}=4.710914 \times 10^{-1}, \mathrm{C}_{1}=1.007833 \times 10^{-2}, \mathrm{C}_{2}=3.8484723$, $\mathrm{C}_{3}=1.200556 \times 10^{3}[18]$.

Considering the GDD caused by both of the prism pair and the $\mathrm{CaF}_{2}$ plate, we have calculated the GDD of the WL seed pulse changing the prism insertion amount putting the 
prism apex at $(\mathrm{x}, \mathrm{y})=(250 \mathrm{~mm},-290 \mathrm{~mm}),(250 \mathrm{~mm},-292 \mathrm{~mm})$, and $(250 \mathrm{~mm},-294 \mathrm{~mm})$ as shown in Figs. 2(b), (c), and (d), respectively.

The WL pulse generated in the $\mathrm{CaF}_{2}$ plate has broadband visible bandwidth extending from 500 to $750 \mathrm{~nm}$. When the WL pulse comes out from the $\mathrm{CaF}_{2}$ plate, the WL pulse has positive chirp experiencing the material dispersion of the $\mathrm{CaF}_{2}$ plate. Meanwhile, the prism pair can give a negative chirp to the positively chirped WL pulse for chirp compensation. Even though the amount of the negative chirp is tunable by changing the insertion amount of the prism pair, the remaining high order chirp cannot be compensated by the prism pair (see Figs. 2(b-d)). The seed pulse with high-order chirp can still be amplified in broadband gainwidth of the NOPA as far as the SH pump pulse duration is stretched longer than the WL seed pulse duration, and this is also one of the reasons why the glass block was inserted in the light path of the pump of NOPA.

In the following sections, 3-2 and 3-3, we have operated the CEP-locked NOPA system compressing the WL seed pulse duration as far as possible, which corresponds to the case shown in Fig. 2 (c). In section 3-2, we have overlapped the SH pump pulse with the center wavelength part of the WL seed pulse to obtain broadest amplification bandwidth for further spectral modification of the gain band (and CEP-locked idler spectrum). In section 3-3, the delay of the SH pump pulse was adjusted to overlap the edge spectrum component of the WL seed to obtain narrowband double peak amplification band (and double color CEP-locked idler pulse).

\subsection{Multi-color CEP locked pulse generation by spatial filter}

In the previous study [12], we have used a custom-made chirp mirror pair to compress the WL seed pulse as transform-limited and most of the spectral component of the WL seed pulse was amplified in broadband from $500 \mathrm{~nm}$ to $800 \mathrm{~nm}$. The broadband signal pulse was generated with idler pulse, whose spectrum is extending from $2000 \mathrm{~nm}$ to $800 \mathrm{~nm}$.

In the present study, the WL pulse duration was roughly compressed by a prism pair. As seen from the GDD curve of Fig. 2(c), the WL seed pulse is estimated to have small chirp around $610 \mathrm{~nm}$. We have adjusted the delay of the SH pump pulse to overlap the SH pump pulse with the WL seed pulse at its spectrum component of $610 \mathrm{~nm}$, and obtained broadest gain in the current configuration of NOPA. Figure 3(a) shows the broadband idler spectrum obtained in the NOPA.

(a)

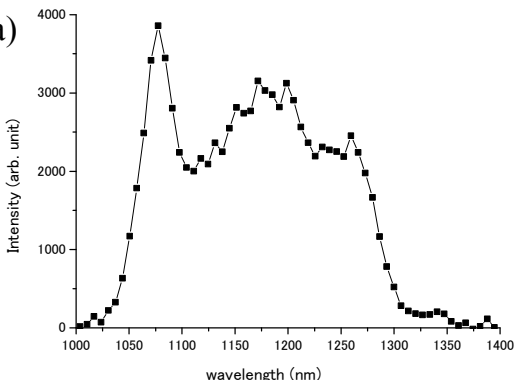

(b)

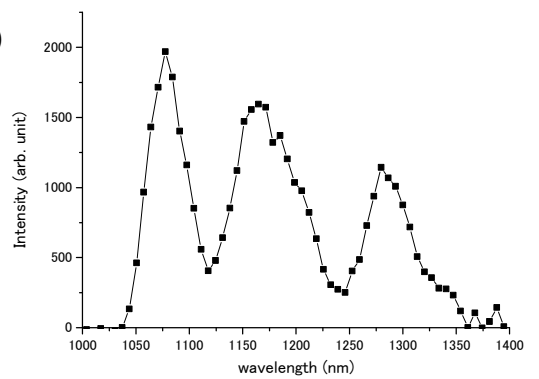

Fig. 3. Spectra of the CEP-locked idler pulse (a) with broadband, and (b) with three peaks obtained by inserting a spatial filter in the spatially dispersed NOPA seed beam.

The idler spectrum obtained in the present work is narrower than that obtained in the previous work [12]. The reason is that the present work used a prism pair to roughly compress the WL seed pulse except for high order chirp introduced by the $\mathrm{CaF}_{2}$ plate and the prism pair. The high order chirp remaining in the WL seed pulse makes its pulse duration longer than that 
of SH pump pulse even though the SH pump pulse duration was stretched by the $40 \mathrm{~mm}$ length glass block.

After obtaining the broadband CEP-locked idler spectrum shown in Fig. 3(a), we have inserted the spatial filter in the light path of the seed beam as shown in Fig. 1. The spatial filter modified the seed spectrum to have three peaks. Amplifying the three-peak seed spectrum in NOPA, the CEP-locked idler pulse also has three peaks in its spectrum (see Fig. $3(b))$.

In the present work, the high order chirp remained in the WL seed pulse was required to generate color-tunable double color CEP-locked idler pulse, which is going to be discussed in the following section. And the spectrum bandwidth of the CEP-locked idler was still enough to demonstrate triple color CEP-locked pulse generation. As a future study, we consider to replace the prism pair with a pulse compression system made of a diffraction grating and a flexible mirror [19], which allows us to compress the WL seed pulse up to high order chirp. Compressing the WL seed pulse as transform-limited pulse, we will obtain ultra broadband CEP-locked idler pulse for ten or more colors of CEP-locked pulse idler pulse.

\section{3-3 Double color CEP locked pulse generation by non-linear chirp}

In this section, we propose and demonstrate another scheme to generate multi-color CEP locked pulse, taking advantage of the high order chirp in the WL seed pulse. Reference 20 shows theoretical support for the passive CEP stabilization including detail derivations of the equations. Generalizing the equations shown in the reference, the equations for multi-color CEP-locked NOPA idler were obtained as shown below.

We assume that three linearly polarized quasi-monochromatic plane waves collinearly propagate in $\mathrm{z}$ direction for the optical parametric amplification (OPA) via three-wave mixing. The double-color seed beam is thought to be a linear superposition of $1^{\text {st }}$-color seed beam and $2^{\text {nd }}$-color seed beam. The $\mathrm{n}^{\text {th }}$-color seed beam generates the $\mathrm{n}^{\text {th }}$-color idler beam in the OPA pumped by the pump beam. Energy conservation rule in the OPA process requires to satisfy $\omega_{p}=\omega_{s n}+\omega_{i n}$, where the subscripts of $p$, sn, in, stand for pump, $n^{\text {th }}$-color seed, and $n^{\text {th }}$-color idler $(n=1,2)$, respectively. Three coupled equations for the real amplitude of respective waves, $A_{m}(m=p$, sn, in; $n=1,2)$, can be obtained from Eqs. A3 of ref. 20 replacing their subscripts of 1,2, 3 with sn, in, $p$, respectively:

$$
\frac{d A_{s n}}{d z}+\alpha_{s n} A_{s n}=\sigma_{s n} A_{p} A_{i n}^{*} \sin \Psi,
$$

(1)

4

$$
\begin{aligned}
& \frac{d A_{i n}}{d z}+\alpha_{i n} A_{i n}=\sigma_{i n} A_{p} A_{s n}^{*} \sin \Psi, \\
& \frac{d A_{p}}{d z}+\alpha_{p} A_{p}=-\sigma_{p} A_{s n} A_{i n} \sin \Psi,
\end{aligned}
$$$$
\frac{d \Psi}{d z}=\Delta k-\left(\sigma_{s n} \frac{A_{i n} A_{p}}{A_{s n}}+\sigma_{i n} \frac{A_{s n} A_{p}}{A_{i n}}-\sigma_{p} \frac{A_{s n} A_{i n}}{A_{p}}\right) \cos \Psi,
$$

where $\Delta k_{k}=k_{p}-k_{\mathrm{gn}}-k_{\mathrm{m}}$, and $\Psi=\phi_{p}-\phi_{s n}-\phi_{i n}-\Delta k \cdot z$ are the phase mismatch and the generalized phase, respectively. The parameters of $\phi_{m}, \alpha_{m}$, and $\sigma_{m}(m=p, s n, i n ; n=1,2)$ are the phases of the respective waves, loss coupling coefficients, and nonlinear coupling coefficients, respectively.

When we assume perfect phase match $(\Delta k \cdot z=0)$ and no loss $\left(\alpha_{m}=0\right)$, Eqs. 2-5 give simplified solutions. In case of parametric amplification process $\left(A_{i n}(z=0)=0\right)$, the generalized phase becomes $\Psi=\pi / 2$ resulting in highest value of $A_{\text {in }}$ after the parametric amplification process. Therefore, $\phi_{i n}=\phi_{p}-\phi_{s n}-\pi / 2$. 
In the work of Warren Warren et. al. [22], the generation and amplification of ultrashort shaped pulse were performed in the visible region by a two-stage NOPA. The spectral amplitude modulation of the shaped pulse was well preserved in the NOPA process in their report. However, there can be strong inter-pulse coupling when the amplification gain is not saturated in the NOPA.

Equations 2-5 show that in general case of multi-color amplification the pump power loss in amplification of one color of the seed pulse decreases the amplification gain of the other colors of the seed pulse, and vice versa. In the present study, the amplification gain was saturated and the output of NOPA has a saturated constant value being irrelevant to the number of the colors.

When the pump power is lowered, the amplification gain in the NOPA is not saturated and the multi-color CEP-locked idler starts to show strong inter-pulse coupling. In the present scheme, the strong inter-pulse coupling is not avoidable when the amplification gain is not saturated. The methodology shown in ref. 22 is thought to be applicable to generate CEPlocked multi-color idler without inter-pulse coupling even when the pump power is lower than saturation level.

The simulation results of group delay in section 3-1 showed that the seed beam has the remaining high order chirp. If the $\mathrm{SH}$ pulse is about $1 \mathrm{ps}$ away from the center wavelength component of the WL pulse, the seed WL pulse can be amplified only around 530nm and 730nm (see Fig. 2(c)). Thus, when the SH pulse does not overlap with the center wavelength component of the WL pulse, the NOPA output will have two peaks in its spectrum. The wavelengths of the two peaks can be tuned by adjusting the delay between the SH pulse and the WL pulse.

To adjust the delay between the SH pulse and the WL pulse, a corner reflector was inserted in the light path of the SH pulse. The position of the corner reflector was scanned by manipulating a micrometer of a manual positioning stage set under the corner reflector. Figures $4(\mathrm{a}-\mathrm{f})$ show the idler spectra observed changing the optical delay between the $\mathrm{SH}$ pulse and the WL pulse at $40 \mu \mathrm{m}$ step. The result shows that we could obtain two-color CEPlocked pulse with wavelength tunability.
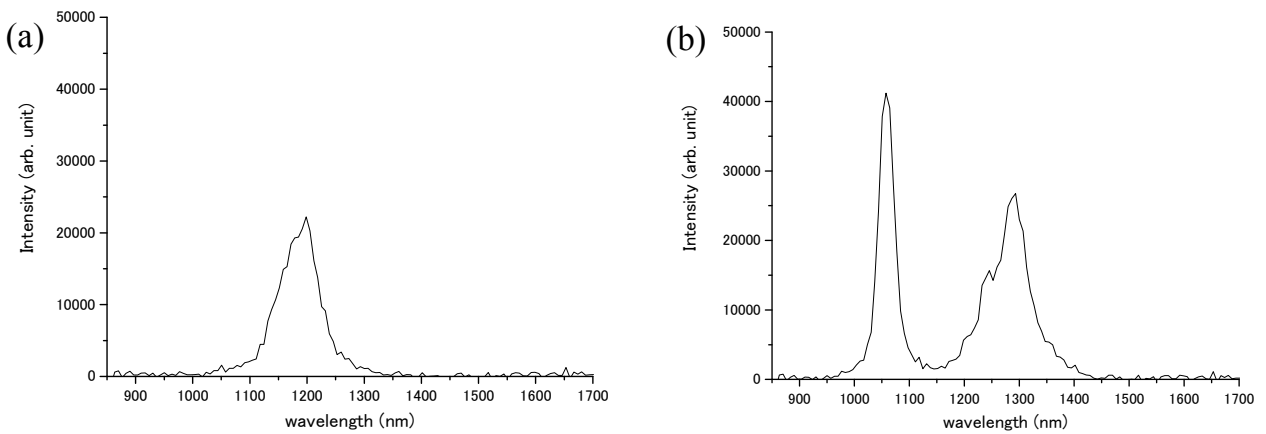

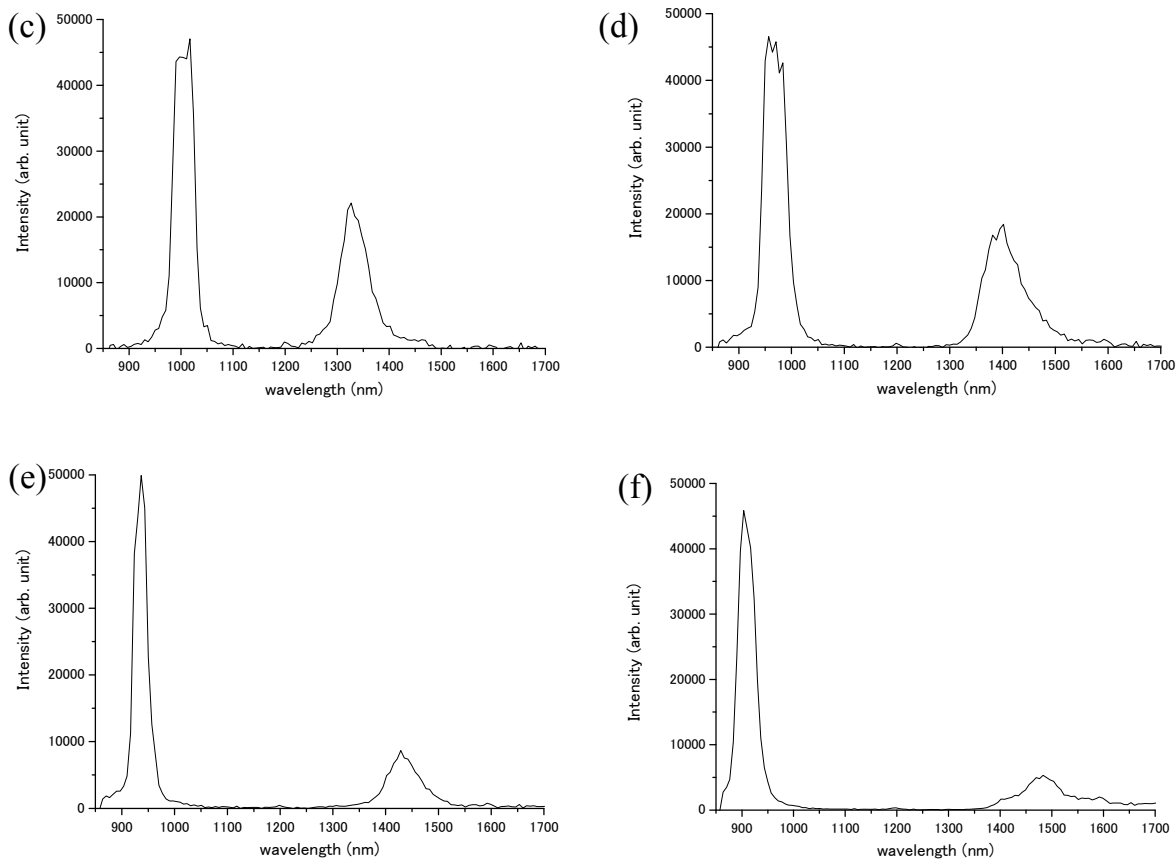

Fig. 4 Spectra of double color CEP locked pulse whose color was tuned by adjusting the delay between the NOPA pump pulse and the NOPA seed pulse. (a)-(f) show the idler spectra observed changing the delay at $40 \mu \mathrm{m}$ step.

In the Figs. 4(a-f), the wavelengths of the double-color CEP-locked pulse was shifted step by step, by changing the optical delay between the SH pulse and the WL pulse at $40 \mu \mathrm{m}$ step. Note that the double color in the spectrum is merged into a single peak in the case of Fig. 4(a). The optical delay can be continuously tuned by shifting the position of the corner reflector, which provides continuous color tunability of the double color CEP-locked pulse.

The wavelengths of the double color CEP-locked pulse are correlated to each others. The wavelength correlation is caused by the dispersion on the seed pulse. Therefore, the correlation of the wavelength is not fixed but tunable by changing the insertion amount of the prism (see Figs. 2 (b-d)).

The CEP-locked idler pulse from the passive CEP stabilization system using NOPA was applied to optical polling in our previous work [21]. Therefore, one of the possible applications of the double color CEP-locked pulse would be as follows. At first, we prepare a sample which contains various chemicals whose absorption bands do not overlap to each other. When the two colors of the light source are tuned to overlap absorption bands of two chemicals, respectively, irradiation of the two color pulses on the sample causes selective alignment of the two chemicals. Making the two chemicals parallel to each other, interaction strength and reactivity between the selected two chemicals is thought to be modified.

In Figs. 2(b-d), the non-linear chirp of the seed light was calculated by the lay tracing simulation. Here, we experimentally estimated the non-linear chirp of the seed pulse from the idler spectra shown in Figs. 4(a-f) and the result is plotted in Fig. 5(a). The delay zero was set to be zero at $540 \mathrm{~nm}$ as same as the ray tracing simulation results of Figs. 2(b-d).

The pulse duration of the pump pulse of NOPA was expected to be $\sim 220 \mathrm{fs}$ from the spectrum of the pump pulse and the material dispersion of the fused silica glass in the optical 
path of the pump pulse. The measured chirp of the seed pulse (see Fig. 5(a)) and the spectral width of the double peaks shown in Figs. 4(a-f) were used to estimate the pump pulse duration of NOPA as $185 \pm 66 \mathrm{fs}$, which agrees with the expected value of $220 \mathrm{fs}$.

For each spectrum of Figs. 4(a-f), we have calculated the duration of the transform limited pulse for each color of the two peaks of the idler spectra (see Fig. 5(b)). The total power of the double color idler pulse was measured to be $\sim 0.8 \mathrm{~mW}$ from the measured power of the signal pulse. When we need to increase the pulse energy of the double-color CEPlocked pulse, the following two possible schemes can be used. First scheme is to put the BBO crystal for NOPA away from the focus of the pump and seed pulses, thus higher pump power can be used for the NOPA process avoiding loss caused by self-phase modulation in the BBO crystal. Second scheme is to implement $2^{\text {nd }}$ amplification stage of the NOPA reflecting back the pump and idler pulses into the BBO crystal by spherical mirrors.
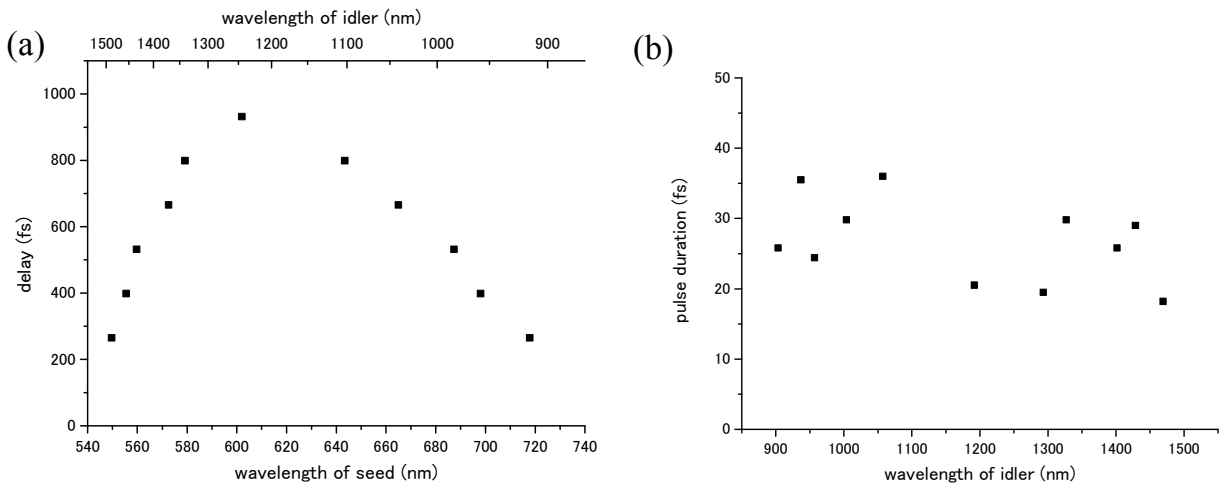

14

15

16

17

18

19

20

21

22

23

24

25

26

27

28

Fig. 5 (a) Chirp of the seed pulse estimated from the wavelengths of the double color idler spectra shown in Figs. 4(a-f). (b) Duration of the transform limited pulse for each color of the two peaks of the idler spectra shown in Figs. 4(a-f).

Then we have adjusted the insertion amount of prism and optical delay of the pump pulse to generate double color idler spectrum with peaks at $800 \mathrm{~nm}$ and $1600 \mathrm{~nm}$. The stability of the CEP was measured by the interference at the wavelength of $800 \mathrm{~nm}$ between the idler $(800 \mathrm{~nm})$ and the second harmonic of the idler $(1600 \mathrm{~nm} / 2=800 \mathrm{~nm})$. The second harmonic was also generated from the BBO crystal of NOPA, which also phase-matches for the second harmonic generation. The observed stability of $\sim 0.54 \mathrm{rad}$ was lower than the previous work $(0.18 \mathrm{rad})[9]$. The difference of stability is thought to be because the optical path length of the seed pulse is longer in the present optical setup to insert prism pair than in the previous optical setup.

\section{Conclusion}

The SH pulse was generated and used as the pump pulse of the NOPA. Focusing a portion of the $\mathrm{SH}$ pulse into a $\mathrm{CaF}_{2}$ plate, the WL pulse was generated to be used as the seed pulse of the NOPA. The WL seed pulse and the SH pump pulse share the same instability of CEP. The idler pulse of the NOPA is generated by difference frequency generation between the SH pump pulse and the WL seed pulse, therefore the idler pulse has no instability in its CEP cancelling out the CEP instability as known from our previous study [9]. The present work proposed and demonstrated two schemes to generate multi-color CEP-locked pulse. 
In the first scheme, we have modified the seed spectrum inserting a prism compressor and a spatial filter in the light path of the seed beam. The three color seed beam was amplified in the NOPA to obtain the three-color idler beam whose CEP is self-stabilized.

In the second scheme, we have adjusted the optical delay between the SH pump pulse and the WL seed pulse to obtain two-color CEP-locked idler pulse whose wavelength is tunable. The two color amplification of the seed (and idler) beam was caused by the high order chirp introduced by the prism compressor in the light path of the WL seed beam.

Meanwhile, the multi-color CEP-locked laser pulse can be generated using other methods inserting a spatial filter for the angularly dispersed idler or inserting a spectral filter in the idler path. The schemes demonstrated in the present work are thought to have the following advantage compared with those methods with idler spatial or spectral filter.

When the spatial or spectral filter is inserted in the idler path keeping the seed spectrum, the pump power in the NOPA is used to amplify all of the broadband phase-matched bandwidth of the seed spectrum even though the simplification is necessary only for the certain multi-color peaks in the spectrum. In the present method, the pump power amplifies only the required multi-color peaks without wasting the pump power for the amplification of unrequested spectral bands, which reduces pump power requirement.

As one of the possible future applications of the present light source, the multi-color CEP-locked pulses are considered to be the potential light sources for quantum control of chemicals.

\section{Acknowledgments}

A.Y. was supported by the National Science Council of Taiwan (NSC99-2923-M-009-004MY3) under FWF (Austria) \& NSC (Taiwan, R.O.C.) bilateral cooperation project. A.Y. and T.K. received a grant from the Ministry of Education, Aim for the Top University Project (MOE ATU) at National Chiao Tung University (NCTU). We also thank the Core Research for Evolutional Science and Technology (CREST) program of the Japan Science and Technology Agency (JST).

\section{References and links}

1. A. Baltuška et al., Nature 421 (2003) 611-615.

2. D. J. Jones et al., Science 288 (2000) 635-639.

3. Y. Kobayashi, K. Torizuka, Opt. Lett. 26 (2001) 1295-1297.

4. V. Roudneve, B. D. Esry, I. Ben-Itzhak, Phys. Rev. Lett. 93 (2004) 163601.

5. M. F. Kling et al., Science 312 (2006) 246-248.

6. C. Day, Phys. Today 59(6) (2006) 13-15.

7. H. R. Telle et al. Appl. Phys. B. 69 (1999) 327-332.

8. A. Apolonski et al. Phys. Rev. Lett. 85 (2000) 740-743.

9. A. Baltuska, T. Fuji, T. Kobayashi, Phys. Rev. Lett. 88 (2002) 133901.

10. T. Wilhelm, J. Piel, E. Riedle, Opt. Lett. 22 (1997) 1494-1496.

11. G. Cerullo, M. Nisoli, S. Stagira, S. De Silvestri, Opt. Lett. 23 (1998) 1283-1285.

12. A. Shirakawa, I. Sakane, M. Takasaka, T. Kobayashi, Appl. Phys. Lett. 74 (1999) 2268-2270.

13. Q. Ding, K. Meng, H. Yang, S. Wang, Q. Gong, Opt. Comm. 284 (2011) 3110-3113.

14. P.F. Wei, J. Liu, C. Li, Opt. Comm. 286 (2013) 334-338.

15. S. Du, D. Zhang, Y. Shi, Q. Li, B. Feng, X. Hang, Y. Weng, J.-Y. Zhang, Opt. Comm. 282 (2009) 18841887.

16. P. Kumbhakar, T. Kobayashi, Opt. Comm. 277 (2007) 205-208.

17. D. Bulton, "Approximate RGB values for visible wavelengths," (1996). http://www.physics.sfasu.edu/astro/color/spectra.html.

18. I. H. Malitson, Appl. Opt. 2 (1963) 1103-1107.

19. A. Baltuska, T. Kobayashi, Appl. Phys. B 75 (2002) 427-443.

20. G. Cerullo, A. Baltuska, O. D. Mucke, Cvozzi, Laser Photonics Rev. (2010) 1-29. 
1 21. K. Okamura, Opt. Comm. 281 (2008) 5870-5873.

2 22. H.-S. Tan, W. S. Warren, E. Schreiber, Opt. Lett. 26 (2001) 1812-1814.

3 